\title{
Aristotle's Syllogistic as a Deductive System
}

\author{
Piotr Kulicki@
}

Institute of Philosophy, The John Paul II Catholic University of Lublin, 20-950 Lublin, Poland; kulicki@kul.pl

Received: 9 April 2020; Accepted: 15 May 2020; Published: 19 May 2020

\begin{abstract}
Aristotle's syllogistic is the first ever deductive system. After centuries, Aristotle's ideas are still interesting for logicians who develop Aristotle's work and draw inspiration from his results and even more from his methods. In the paper we discuss the essential elements of the Aristotelian system of syllogistic and Łukasiewicz's reconstruction of it based on the tools of modern formal logic. We pay special attention to the notion of completeness of a deductive system as discussed by both authors. We describe in detail how completeness can be defined and proved with the use of an axiomatic refutation system. Finally, we apply this methodology to different axiomatizations of syllogistic presented by Łukasiewicz, Lemmon and Shepherdson.
\end{abstract}

Keywords: Aristotle's logic; syllogistic; Jan Łukasiewicz; axiomatic system; axiomatic refutation; completeness

\section{Introduction}

Deductive systems of different kinds are the heart of contemporary logic. One could even state that logic itself, as it is understood nowadays, is just a collection of deductive systems appropriate for different kinds of reasoning. Even when ways of reasoning that are usually distinguished from deduction, such as induction or abduction, are considered, they are finally presented in a deduction-like form of a strict system. The theory and methodology of deductive systems is established and well developed, and so is the folklore spread through the community of logicians.

While discussing deductive systems in contemporary logic, it is however still interesting to look back to the roots of Western logic and compare what we have achieved today with the legacy of Aristotle. Somehow surprisingly, we can find many features of today's mature deductive systems in his system of syllogistic. Robin Smith in his entry in the Stanford Encyclopedia of Philosophy [1] notices that "scholars trained in modern formal techniques have come to view Aristotle with new respect, not so much for the correctness of his results as for the remarkable similarity in spirit between much of his work and modern logic. As Jonathan Lear has put it, 'Aristotle shares with modern logicians a fundamental interest in metatheory': his primary goal is not to offer a practical guide to argumentation but to study the properties of inferential systems themselves." Thus, analysing Aristotle's syllogistic allows us to reflect on the most essential features of a deductive system and abstract them from their exact content, context and the terminology used.

No wonder that in recent decades we can observe a significant interest in the logical works of Aristotle. Klaus Glashoff in 2005 [2] (p. 949) stated that "[u]nlike several decades ago, Aristotelian logic meets with growing interest today. Not only philosophers, but also specialists in information and communication theory employ ideas which can be explicitly traced back to Aristotle's work on categories and syllogisms. [...] Independently of these rather recent developments, there has been a renewed interest in matters of formalization of Aristotelian logic by a small group of logicians, philosophers and philologists." Since then, many new works have been published either directly on the writings of Aristotle [3-5] or on extensions or technical aspects of his syllogistic [6-19], to mention only a few. 
After Aristotle, syllogistic was for many centuries the dominant form of logic attracting interest of many generations of scholars. There were at least a few important contributions to the theory before the rise of modern mathematical logic in the twentieth century, including the medieval systematisation of traditional syllogistic, several mathematical interpretations of syllogistic presented by Gottfried Wilhelm Leibniz and the diagrammatic approach to the theory introduced by Leonard Euler and John Venn. In this paper we are, however, interested mostly in modern reconstructions of syllogistic starting from the works of Jan Łukasiewicz and some of the ideas inspired by Aristotle presented in this context.

We will start our considerations with some remarks on the original presentation of syllogistic given by Aristotle mainly to trace his methodology of deductive systems. Then, we will look at the system presented by Łukasiewicz. From the perspective of almost a century we trace and assess the choices he made while formalizing syllogistic. We will be especially interested in the way Łukasiewicz developed the Aristotelian discussion of the completeness of the system of syllogistic. Moreover, we will compare this approach with theory and practice of completeness investigations in contemporary logic. Finally we will present how Łukasiewicz's methodology works on the several variants of the system of syllogistic.

The technical results presented in the paper are not novel. The most interesting from the technical point of view is perhaps the refutation counterpart of Shepherdson's axiomatization of syllogistic. The main contribution of the paper lies in its methodological discussion of the issue of correctness of a deductive system. The paper is also rich in references covering sources that present different attempts at the formalization of syllogistic, as well as selected recent works on the subject.

\section{Original Presentation}

To obtain the right perspective in order to discuss some details of the modern formalizations of syllogistic let us start from a few remarks on its original, Aristotelian presentation. Innocenty M. Bocheński expressed a very strong, but in principle right, opinion on its role in the history of thought that " $\mathrm{t}]$ he assertoric syllogism is probably the most important discovery in all the history of formal logic, for it is not only the first formal theory with variables, but it is also the first axiomatic system ever constructed" [20] (p. 46). This claim takes into account the significance of the Aristotelian system not only for logic. Formal theories and formal modelling are ubiquitous in modern science. Mathematics and mathematically founded physics have been using these tools for the longest time but many other disciplines of natural and social science build their own formalized theories which share the same crucial features. It was Euclidean geometry that in the modern era gained the position of the icon of a deductive system (c.f. famous Spinoza's more geometrico but it was syllogistic that earlier had set the standard and prepared the basic conceptual framework for formal techniques in science.

Bocheński justifying his claim on the importance of syllogistic mentioned two issues: the use of variables and the form of an axiomatic system. While the former is simple, understanding the latter requires a reflection on what an axiomatic system is. To acknowledge that a theory forms an axiomatic system two things are required. One is a division of the elements of the system into two groups: axioms and theorems. Some propositions (let us at this point skip the issue, to which we will come back in the following section, of whether syllogisms are indeed propositions, since the same construction can be designed for objects other than propositions, like valid rules or designated modes of reasoning) are treated in a special way and are accepted as axioms and other propositions are derived from them. The other requirement concerns the relation between axioms and theorems. Theorems are derived and the derivation must be deductive. This is the point where maturity of deduction methodology can be observed. In mature systems rules of deduction are explicit and formal.

In the Aristotelian presentation of syllogistic the syllogisms of the first figure are perfect (they are axioms) and the syllogisms of the two other figures are imperfect (i.e., derived from axioms). Bocheński [20] (pp. 46-47) points out three rules of deduction used by Aristotle in his axiomatic system of syllogistic: the direct reduction, the reductio ad impossible and the ecthesis. These rules are deductively 
valid and recognized in contemporary logic. In modern terminology we can call the direct reduction strengthening of a premise, the reductio ad impossible — transposition, and the ecthesis-reasoning by example. Since we are interested only in the fact of axiomatization and the level of formalization we are not going to present the precise formulation of rules and details of derivations here (for the reconstructions of proofs of all syllogism see [20] (pp. 49-54)).

In a series of loose notes placed throughout Posterior Analytics, we can also find general rules of construction of an axiomatic theory. Bocheński reconstructs them in the following way [20] (p. 46):

1. there must be some undemonstrated claims: axioms, and other claims: theorems are deduced ([21], 72b),

2. axioms must be intuitively evident ([21], 99b),

3. the number of steps of deduction in proofs of theorems must be finite ([21], 81b).

Aristotle's approach to axiomatization is similar but not identical to the contemporary one. The main difference lies in the above point 2 regarding axioms. Conditions such as self-evidence, certainty and ontological priority are no longer imposed on them. An axiom differs from other statements of a system only in the fact that it is not derived (c.f. [22] (pp. 70-71)). There are different axiomatizations of the same theories and they are equally correct provided they define the same set of accepted objects. Still some choices of axioms may be evaluated higher than others. What are the criteria applied by contemporary logicians here? The answer is not straightforward. Surely, most of them are not strict and formal. Some of them are similar to what Aristotle required. Sometimes we value higher axioms that are intuitively clear or self-evident. Similar to these criteria is the simplicity of axioms, which is sometimes stressed as an advantage. On the other hand, sometimes axiomatizations with a smaller number of axioms are evaluated higher.

There are some more metalogical notions whose presence (or at least traces) in the Aristotelian system of syllogistic is pointed out by some authors. We will discuss the notion of completeness in detail in the following sections, now let us just briefly mention the notion of compactness.

The issue of compactness of Aristotle's syllogistic was raised by Lear [23]. He claimed that in Posterior Analytics I.19-22 Aristotle discusses a proof-theoretic analogue of compactness. Compactness itself is a model theoretical property of a system stating that if a proposition $\alpha$ is a semantic consequence of an infinite set of propositions $\varphi$, then there exists a finite set $\varphi_{1} \subset \varphi$ such that $\alpha$ is a semantic consequence of $\varphi_{1}$. What is then the proof-theoretic analogue of compactness? It is a property stating that every demonstrable conclusion can be demonstrated from finitely many premises. In other words, there are no valid ways of deductive reasoning that effectively use infinitely many premises.

The question arises whether what Aristotle discusses is really related to compactness in the sense used in contemporary metalogic or it is just a misinterpretation of Aristotle. The second opinion is presented by Michael Scanlan [24], who states that introducing compactness in the context of syllogistic is anachronistic since Aristotle did not use model theory at all. An interesting and balanced discussion of the issue is presented by Adam Crager in [4]. In the context of the present paper it is enough to ascertain that some contemporary logicians want to find traces of modern logical ideas in Aristotelian works even if they are not quite clear there, and that these logicians might be right.

\section{Lukasiewicz's Reconstruction of Syllogistic: Formalization Choices}

\subsection{Preliminaries}

It is hard to tell whether Łukasiewicz was aware of the different possibilities he could use when he was formalizing syllogistic using the tools of modern formal logic. "the" in the title of his book: Aristotle's Syllogistic from the Standpoint of Modern Formal Logic may suggest that in his opinion his point of view concerning the theory was the only one.

Now, taking into account later works on syllogistic we can see that it is not that simple. There are many possible formal tools that can be applied to construct a system of syllogistic and many variants 
of the content of the theory. Looking from today's perspective the most fundamental decision is the choice of a kind of object a syllogism should be. In the later literature (see e.g., [25,26]) at least three interpretations of a (correct) syllogism are discussed: (1) valid premise-conclusion argument, (2) true proposition or (3) cogent argumentation or deduction. In terms of the formal structure that leads to two clear possibilities: inference rules for (1) and implication propositions for (2). Interpretation (3) requires a less direct formal account of syllogism.

Łukasiewicz constructed a theory where syllogisms are represented as propositions. This approach seems to be in accordance with the spirit of the 30s in logic. The hype was for axiomatisation in, what we would call now, the Hilbert style. Natural deduction, being an alternative to it, had just been invented by Gentzen and Jaśkowski and only budding. It is less known that Gödel in his Notre Dame lectures in 1939 [27] also presented a formalization of syllogistic with the use of mathematical logic and his system was constructed in a way similar to Łukasiewicz's system. The main difference was in the choice of axioms and in the fact that while Łukasiewicz presented a full-fledged theory, Gödel presented only a sketch.

Another important issue where approaches to syllogistic may vary is connected with the sort of names that can be used within categorical sentences that are the components of syllogisms. Two distinctions are relevant here for individual/common names and empty/nonempty ones. This issue was also discussed extensively after Łukasiewicz and different proposals are now available here. In the following sections we will discuss Łukasiewicz's approach in detail.

\subsection{Axiomatic Theory Based on the Classical Propositional Logic}

What is shared by all the aforementioned interpretations of Aristotelian logic is that the purpose of syllogistic is to study reasoning in which categorical propositions are both premises and conclusions. As we have mentioned, such reasoning can be formalized with the tools available to modern formal logic, in several ways, for example as sentences of language with the implication structure or as inference rules or schemata.

In the former case, the premises for reasoning can be treated as factors of the conjunction constituting the antecedent of implication, and the conclusion as the consequent of implication. What results are formulas that can be converted into rules in a natural way. To view syllogisms as such implications requires an interpretation of implication and conjunction. Łukasiewicz adopted the simplest solution available for him, where these operators are taken from the classical propositional calculus. The classical interpretation of operators is, however, by no means obvious. The definition of syllogism itself, as derived from Prior Analytics: "[a] syllogism is an argument in which, certain things being posited, something other then what was laid down results by necessity because these things are so", [21] $(24 \mathrm{~b}, 20)$ suggests two features of syllogisms that the classical calculus ignores-non-tautologicality "something other then what was laid down" and relevance: "because these things are so" (see e.g., [3] for a discussion of the issue of relevance).

Łukasiewicz went further to assume that syllogistic is built over the whole classical propositional calculus and thus allows structures other than those in the form of syllogism. In this way, the direct relationship with rules is lost. This element of his approach to Aristotle's syllogistic seems to be particularly controversial.

Therefore, to provide a better understanding of the essence of Łukasiewicz's approach to syllogistic, three elements can be separated: (1) the formalization of reasoning by sentences of language with an implication structure, (2) the use of the classical understanding of propositional operators, (3) the use of propositional calculus operators in any configuration to build complex formulas.

Łukasiewicz's approach was strongly criticized by John Corcoran [28,29]. His criticism concerned mainly point (1) above. Instead, Corcoran proposed to formalize syllogisms as rules within a system of natural deduction. From the further perspective, however, the difference between the two approaches is not that essential. When propositions in the form of implication are considered, there is a close relation between the truth of sentences and the soundness of inference rules. True implications are the 
basis of correct rules, and correct rules can be transformed into corresponding true sentences. Such a proposition-rule duality of implications reveals itself especially in the context of logic programming. Logic programs are sets of Horn clauses. In a declarative interpretation clauses are implications in which ancetedents are conjunctions of atoms and consequents are atoms. In a procedural interpretation they are rules with multiple premises that are triggered in certain situations. It is easy to see that syllogisms have the same structure and therefore they also can be interpreted dually.

Indeed, the other aspects of Łukasiewicz's approach seems to be more controversial. Classical propositional calculus is probably not the logic which can adequately describe the Aristotelian way of thinking. Moreover, Aristotle did not use any structures other than standard syllogisms and sorites (syllogism with more than two premises). Again these discrepancies between the Aristotelian theory and its reconstruction by Łukasiewicz does not seriously undermine Łukasiewicz's practice. That is because he does not really make use of propositional logic more than it is necessary to reconstruct arguments confluent with the ones acceptable by Aristotle, mainly proving one syllogisms on the basis of others.

\subsection{Admissible Types of Names}

In logical semiotics, there are two divisions of names that are interesting from the point of view of the formalization of the syllogistic. The first is made by the type of reference and distinguishes between common and proper (individual) names. Common names designate objects because they meet some conditions and can always be linked with appropriate predicates that represent these conditions. Individual names designate specific objects under a language convention. The other division is made by the number of designates and distinguishes between empty names, i.e., having no designates, particular names, i.e., having exactly one designate, and general names, i.e., those having more than one designate. Note that empty and particular names can be common or proper. As an example of a common empty name we can take "unicorn" or "square circle", as an example of a proper empty name we can take "Pegasus" or "Santa Claus".

While building a system of logic of names such as syllogistic one can narrow the range of names that can be used to selected categories based on the above divisions. Such postulates have had various motivations and justifications. In his famous work "On Sense and Reference" [30], Gottlob Frege proposed that empty names be eliminated from the language of science. In his justification, Frege uses reasoning that can be summarized in the claim that the use of names without denotation leads to pointless discussion and manipulation. Aristotle permits names without denotation, assuming that atomic sentences in which such names appear are false. Łukasiewicz's formalization of syllogistic assumes that all names are non-empty. However, many systems built in his style, like [31-34], allow the use of empty names.

Łukasiewicz also eliminates individual names from the language of syllogistic. A similar narrowing can be observed in Peter Geach, who in combining individual and proper names in traditional syllogistic sees an important source of the "corruption of logic" [35]. The position of Aristotle himself on this issue is not quite clear. When he presents valid syllogisms in Prior Analitics (26a-46b) he always uses general names like "animal", "man" and "white". However further in Prior Analitics 47b while discussing some invalid forms, where "no syllogism is possible" he puts in these forms individual (proper) names: Aristomenes and Miccalus. It is not obvious, and Aristotle does not state clearly, whether the use of proper names is the reason why syllogisms are not possible or it is a coincidence.

The admissibility of propositions in which the same argument appears twice raises yet another type of doubt. In modern logic, such formulas are natural and can be created by substituting the same value (constant or variable) in any expression. In his system formalizing the syllogistic of Aristotle,

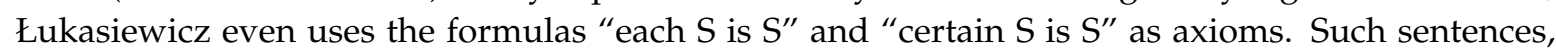
however, do not appear in the description of syllogistic modes given by Aristotle. 
This fact can be associated with the requirement mentioned above, according to which syllogism should lead to new knowledge. On its basis, one can derive "something else than assumed", and, on the other hand, what is derived "must result because it was assumed". In this context, the sentences "every $S$ is $S^{\text {" }}$ and "certain $S$ is $S$ " are not useful, because with the normal use of syllogisms nothing new results from them, nor can they constitute new knowledge resulting from certain assumptions. Łukasiewicz does not set out any restrictions on substitution.

\section{Completeness}

The basic criterion for assessing the quality of a formal system is its adequacy with respect to underlying intuitions. Adequacy consists of two properties: soundness and completeness. In the case of an axiomatic system, soundness means that all theorems of the system follow underlying intuitions, and completeness that all formulas that are intuitively accepted are also accepted in the system. In the literature one can find many embodiments of this fundamental intuition which differ in important details. We will cite some of them below. In the first two, completeness directly refers to sets of formulas, and the truth of these formulas (sentences) is adopted as the intuitive acceptance criterion. Kazimierz Ajdukiewicz uses the concept of completeness understood as follows:

"each true sentence that can be formulated in the language of this theory can be proved (unless it is an axiom of this theory) by the means of evidence at its disposal." [36] (p. 215)

Ludwik Borkowski gives the following definitions of completeness:

"The $S$ system is complete if and only if each true expression of the $S$ system is a thesis of the S system." [37] (p. 378)

In metalogical considerations, the classical correspondence concept of truth is usually used. In Ajdukiewicz's formulation it is as follows:

"Any declarative sentence is true when it is just as it says; it is false when it is not what it proclaims." [36] (p. 29)

The above framings have, however, a disadvantage. Not all formal systems that logic deals with refer to truthfulness. An example would be intuitionistic logic, where the goal is to capture what is constructively provable rather than true. However, the same method can still be applied to consider completeness in relation to formal approaches to this type of logic. In general, the concept of completeness in the above approaches may be retained, only the term "true" should be replaced with the term "accepted" or "admissible".

Other definitions associate completeness with sets of inference or reasoning methods and refer to their soundness or reliability, which corresponds to the truthfulness of sentences. Andrzej Grzegorczyk writes about completeness:

"The natural, historical development of logic has indeed led to the creation of such a logic system, which can be proved to contain all logical methods (schemata) of correct inference on any subject. We call this property completeness." [38] (p. 121)

Witold A. Pogorzelski phrases this concept as follows:

"The problem of completeness can be formulated as a question of whether all reliable ways of reasoning are actually based on the laws of formal logic." [39] (p. 366)

As we noted earlier, there is a close relationship between the truth (acceptance) of sentences and the soundness of reasoning (rules of inference). True sentences can in fact form the basis for the construction of correct reasoning, and correct reasoning can be transformed into corresponding true sentences. This fact allows us to assume that all the above definitions express in their own way the 
same intuition, which does not give rise to controversy. In all definitions, completeness is semantic in the sense that it refers a formal system to something external, to reality, or at least to a way of thinking about reality.

How to verify which sentences are true remains a problem, and in particular, how to do it precisely enough to be able to use this validation in logical research. Most often formal models are used for this purpose and truthfulness is defined as truthfulness in a model, which is defined by the formal conditions imposed on objects in the model. As a result, in practice the truthfulness of sentences is usually equated with their truthfulness in a formal model or a class of models. Consequently models directly appear in the definition of completeness, as in the definition below from the Small Encyclopedia of Logic edited by Witold Marciszewski:

"The deductive logic system is complete if and only if all sentences that are true sentences in each model can be derived from its axioms." [40] (p. 236)

However, by adopting this position, we give up the semantic character of completeness. We consider the mutual relations between the two formal systems, i.e., the axiomatic system and the system defining the formal model. Two formal approaches undoubtedly give a more complete picture of a formal theory, but it does not connect the theory with reality. The problem of completeness of the axiomatic system with respect to the underlying intuitions is not solved by demonstrating completeness in relation to a model, but is only put aside. Another problem arises, one of adequacy of the formal model in relation to reality or the way of thinking which the formal system under consideration is to capture. Sometimes a model theoretical structure is intuitive, but in some cases, as relevance logic, linear logic, or even intuitionistic logic, a proof-theoretical approach is much closer to intuitions then models constructed to match the systems.

In the case of syllogistic, set-theoretical models are quite intuitive and seems to be natural, especially for contemporary people, who are accustomed to thinking in terms of sets from kindergarten. However, there are reasons to consider a theory of syllogism that is not dependent on set-theoretical models. One of them is historical: Aristotle himself did not know set theory. Thus, it is good to be able to conduct metalogical considerations concerning syllogistic without sets just to avoid anachronisms. The other reason is that some researchers claim that set-theoretical approach used in modern logic does not fit to the way we use natural language and use alternative approaches like Leśniewski's ontology [41].

Following and referring to Aristotle, Łukasiewicz proposed a different solution. In Prior Analytics Aristotle shows that syllogistic schemata other than the syllogisms that he accepted should not be accepted. In this way, he proves completeness of his system of syllogistic. He considers all possible schemata with two premises belonging to each of the three figures. In most cases, he justifies the rejection of a schema by providing a counter-example, as in the following passage:

"Nor will there ever be a syllogism if both intervals are particular, whether positive or privative, or if one is stated positively, the other privatively, or one indeterminate, the other determinate, or both indeterminate. Common terms for all cases: animal, white, horse; animal, white, stone." [21], (26b)

Showing examples falsifying all unacceptable formulas is labour-intensive, and in many cases impossible due to their unlimited number, e.g., if one were to consider reasoning with any number of premises (factors in the antecedent of implications). Already in Aristotle, however, one can find a hint regarding a different way of rejecting such formulas, which Łukasiewicz extracts and expands. The following text occurs in Aristotle:

"For since it is true that $M$ does not belong to some $X$ even if it belongs to none, and there was no syllogism when it belonged to none, it is evident that there will not be one in this case either." [21], (27b) 
Out of this short note of Aristotle, Łukasiewicz derived an idea of axiomatic refutation that was first further developed by Słupecki and his collaborators and then entered into the wider logic community. A substantial theory concerning the logic of rejected propositions is presented in [42]. Recently the achievements in the field were recapitulated in [43] and the Łukasiewicz-Słupecki approach to the issue was discussed in [44].

The basic ideas of Łukasiewicz are as follows. In addition to usual axioms and rules, rejected axioms and rules of refutation are introduced. Rejected axioms should not be valid and rules of refutation also produce non-valid formulas. A system is said to be refutationaly complete if each formula of its language is either a theorem or a rejected formula. In his writings Łukasiewicz, and also Słupecki, stressed that under certain conditions refutationaly complete systems are decidable.

In the opinion of the Author of the present paper, even more interesting is the argument that refutationaly complete systems are adequate (sound and complete) in the very basic sense of adequacy discussed above. In the following section we will present how refutation works in Łukasiewicz's system and in some other axiomatizations of syllogistic constructed in his style.

\section{Axiomatic Systems of Syllogistic with Refutation Counterparts}

The language of all the systems discussed in this section is the same. It contains name variables $S, P, M, N, \ldots$, propositional operators and the two primitive operators $a$ and $i$ specific for syllogistic read in a usual way: $S a P$ is read as "every $S$ is $P$ " and SiP_as "some $S$ s are $P$ s" or "certain $S$ is $P$ ". We will call formulas like $S a P$ and SiP atoms. Formally, a formula of the language can be defined in the following way (using Backus-Naur notation):

$$
\alpha=\operatorname{SaS}|\operatorname{SiS}| \neg \alpha|\alpha \wedge \alpha| \alpha \vee \alpha|\alpha \rightarrow \alpha| \alpha \equiv \alpha
$$

The usual negative syllogistic operators $e$ and $o$ (where $S e P$ is read as "no $S$ is $P$ " and $S o P$-as "some $S$ s are not $P \mathrm{~S}$ " or "certain $S$ in not $P$ ") can be defined as negations of the primitive operators:

$$
\begin{aligned}
& S e P \triangleq \neg S i P, \\
& S o P \triangleq \neg S a P .
\end{aligned}
$$

In all the systems any substitution of a classical tautology is an axiom. The common derivation rules are Modus Ponens MP and substitution $S u b$ of the following schemata:

$$
\begin{gathered}
\frac{\vdash \alpha \rightarrow \beta ; \vdash \alpha}{\vdash \beta} \\
\frac{\vdash \alpha}{\vdash e(\alpha)},
\end{gathered}
$$

where $e$ is a substitution for name variables.

\section{1. Łukasiewicz}

Let us start with the Łukasiewicz's system. Its specific axioms of are as follows:

SaS,

SiS,

$$
M a P \wedge S a M \rightarrow S a P,
$$

$$
M a P \wedge M i S \rightarrow \operatorname{SiP}
$$

The negative (rejected) part of the system is defined by the following three rules $(\vdash$ marks an accepted formula and $\dashv$ a rejected one): 
- $\quad$ rejection by detachment $M P^{-1}$.

$$
\frac{\vdash \alpha \rightarrow \beta ; \dashv \beta}{\dashv \alpha}
$$

- $\quad$ rejection by substitution $S u b^{-1}$ :

$$
\frac{\dashv e(\alpha)}{\dashv \alpha},
$$

where $e$ is a substitution for name variables,

- decomposition rule Comp ${ }^{-1}$ :

$$
\frac{\dashv \alpha \rightarrow \beta_{1} ; \ldots ; \dashv \alpha \rightarrow \beta_{n}}{\dashv \alpha \rightarrow \beta_{1} \vee \ldots \vee \beta_{n}}, n \geq 1,
$$

where $\alpha$ is a conjunction of atoms and $\beta_{i}(1 \leq i \leq n)$ are atoms.

The last rule is a variant from [45] of the rule of Słupecki, used by Słupecki, Łukasiewicz and Shepherdson, that reflects in its shape a more general result on Horn theories from [46]

The following formula is the sole rejected axiom:

$$
P a M \wedge S a M \rightarrow S i P .
$$

To see how the axiomatic system works let us give a proof of the following conversion law for general negative sentences (for the application of the laws of propositional calculus we use the abbreviation "PC", to abbreviate substitutions like $e(M)=P$ we will write $M / P$ ):

$$
\mathrm{SeP} \rightarrow \mathrm{PeS}
$$

1. $M a P \wedge M i S \rightarrow \operatorname{SiP} \quad$ axiom (4)

2. $P a P \wedge P i S \rightarrow$ SiP $\quad$ Sub $: 1(M / P)$

3. $\mathrm{PaP} \rightarrow(\mathrm{PiS} \rightarrow \mathrm{SiP}) \quad \mathrm{PC}: 2$

4. $M a M \quad$ axiom (1)

5. $P a P \quad S u b: 4(M / P)$

6. $P i S \rightarrow S i P \quad M P: 3,5$

7. $\neg$ SiP $\rightarrow \neg P i S \quad$ PC: 6

$\mathrm{SeP} \rightarrow \mathrm{PeS} \quad$ definition of $\mathrm{SeP}: 7$

As an example of a negative derivation let us give a refutation of the analogous conversion of general positive sentences:

$$
\mathrm{SaP} \rightarrow \mathrm{PaS}
$$

1. $\vdash M a P \wedge S a M \rightarrow S a P$

axiom (3)

2. $\vdash \mathrm{MaP} \wedge \mathrm{MiS} \rightarrow \mathrm{SiP}$

axiom (4)

3. $\vdash$ SiS

axiom (2)

4. $\vdash \mathrm{SaP} \wedge \mathrm{SiS} \rightarrow \mathrm{SiP}$

Sub: $2(M / S)$

5. $\vdash \mathrm{SiS} \rightarrow(\mathrm{SaP} \rightarrow \mathrm{SiP})$

PC: 4

6. $\quad \vdash \mathrm{SaP} \rightarrow \mathrm{SiP}$

$M P: 5,3$

7. $\vdash M a P \wedge S a M \rightarrow S i P$

PC 1,6

8. $\vdash(\mathrm{MaP} \wedge \mathrm{SaM} \rightarrow \mathrm{SiP}) \rightarrow((\mathrm{PaM} \rightarrow \mathrm{MaP}) \rightarrow(\mathrm{SaM} \wedge \mathrm{PaM} \rightarrow \mathrm{SiP})) \quad \mathrm{PC}$

9. $\vdash(P a M \rightarrow M a P) \rightarrow(S a M \wedge P a M \rightarrow S i P)$

MP $: 8,7$

10. $\dashv P a M \wedge S a M \rightarrow S i P$

11. $\dashv P a M \rightarrow M a P$ rejected axiom (5)

$\dashv \mathrm{SaP} \rightarrow \mathrm{PaS}$ $M P^{-1}: 9,10$ Sub $^{-1}: 12(S / P, P / M)$

Łukasiewicz shows that all the Aristotelian assertoric syllogisms and all the one-premise valid reasoning schemata mentioned by Aristotle have their counterparts in the form of implications provable in Łukasiewicz's system. Moreover, since the system incorporates the whole classical propositional 
calculus, many formulas that are not directly connected to the reasoning schemata discussed by Aristotle, or are not implications e.g.,:

$$
\begin{gathered}
S a P \rightarrow S a P, \\
S i P \vee S o P, \\
\neg(S a P \vee S e P)
\end{gathered}
$$

are provable.

Łukasiewicz's system is refutationally complete, i.e., every formula of the language is either a theorem or can be rejected. The proof of that fact is well known (see $[47,48])$ and we will not repeat it. Let us just mention that the proof relies on the observation that a formula of a form $\alpha \rightarrow S a P$, where $\alpha$ is a conjunction of atoms, is a theorem if and only if $\alpha$ contains a chain connecting $S$ with $P$, and such a formula is rejected whenever it is not a theorem. A chain is a conjunction of the following form:

$$
S a M_{1} \wedge M_{1} a M_{2} \wedge \ldots \wedge \mathcal{M}_{n} a P(n \geq 0) .
$$

The result is then extended to all formulas of the language on the basis of propositional calculus derivations on the accepted side, and the refutations based on the rules $M P^{-1}$ and Comp ${ }^{-1}$ on the rejected side.

\subsection{Lemmon}

Let us now look at systems without restriction to non-empty names. Many different axiomatizations adequate for that idea were introduced. They differ, beyond just the choice of axioms, in two main points. One of them is the set of primitive notions: some of them use the same operators $a$ and $i$ as Łukasiweicz, some other use nominal negation instead of $i$. The other difference is the interpretation of the operator $a$, which can be strong or weak. In both of them, obviously, for $S a P$ to be true $S$ must be contained in $P$, but in the strong interpretation $S$ must be non-empty, while in the weak one it is not so.

The strong interpretation is adopted, among others, by Wedberg, Menne and Lemmon [32,33,49]. We start from it because the refutation part of this variant of theory is much simpler than the one for the weak interpretation. We use a variant of Lemmon's system from [45] with Equations (3) and (4),

$$
\mathrm{SaP} \rightarrow \mathrm{SiP},
$$

and

$$
\mathrm{PiS} \rightarrow \mathrm{SaS}
$$

as axioms.

The refutational counterpart of the system consists of the same rules as for Łukasiewicz's system, and Equation (5) and

$$
\mathrm{PaP} \rightarrow \mathrm{SiS}
$$

as rejected axioms. The proof of refutation completeness of the system similar to the one for Łukasiewicz's system can be found in [50].

\subsection{Shepherdson}

Now we can pass to Shepherdson's system [34], also called the Brentano style syllogistic [51] (p. 311). The speciffic axioms of the system are in Equations (1), (3) and (4),

$$
\mathrm{SiP} \rightarrow \mathrm{SiS},
$$


and

$$
S a P \vee S i S .
$$

Assuming the correctness of Lemmon's system we can obtain correctness result for Shepherdson's by embedding it into Lemmon's. To define the relation between Shepherdson's and Lemmon's systems let us for this purpose distinguish two variants of operator $a_{\text {: }} a_{S}$ and $a_{L}$ occurring in the two systems, respectively. With this convention we can formulate the following equivalences that may be used to mutually define one operator by another:

$$
\begin{gathered}
S a_{S} P \equiv S a_{L} P \vee \neg(S i S), \\
S a_{L} P \equiv S a_{S} P \wedge S i S .
\end{gathered}
$$

Let us, however, introduce the refutational counterpart of Shepherdson's system. The system was first presented in [50] where full proof is given (it is lengthy and laborious but quite predictable). Here we will just sketch the proof and use the final result to comment on the usefulness of the refutation approach for the discussion of completeness.

Let us start with the easier part: the refutation system for the Horn fragment of the Shepherdson's system, i.e., the system with Equations (1), (3), (4) and (9), as axioms.

Here the rules of rejection are the same as in Łukasiewicz's system and the rejected axioms are:

$$
\begin{gathered}
S i S \wedge P i P \wedge S a M \wedge P a M \rightarrow S i P \\
P i P \wedge S a P \rightarrow S i S .
\end{gathered}
$$

The proof of refutation completeness is analogous to the one for Łukasiewicz's system.

Now, let us come back to the full system of Shepherdson, and its refutation counterpart. To define it we will use the rules $M P^{-1}, S u b^{-1}$, as in the Łukasiewicz's system and the following modified version of $\operatorname{Comp}^{-1}, \operatorname{Comp}_{2}^{-1}$ :

$$
\frac{\dashv \alpha \rightarrow \beta_{i} \vee \beta_{j}, \text { for each } \mathrm{i}, \mathrm{j}, \text { such that: } 1 \leq i<j \leq n}{\dashv \alpha \rightarrow \beta_{1} \vee \ldots \vee \beta_{n}}, n \geq 2,
$$

where $\alpha$ is a conjunction of atoms and $\beta_{i}(1 \leq i \leq n)$ are atoms.

The sole rejected axiom is as follows:

$$
M a S \wedge M a P \wedge M a Q \wedge S a R \wedge P a R \wedge R a N \wedge Q a N \wedge S i S \wedge P i P \wedge Q i Q \rightarrow S i P \vee R i Q
$$

The choice of rejected axiom is mainly technical: it is chosen as sufficient to prove completeness. In the following section we will show by giving an example that it is not valid and for that reason it should be rejected. The role of the Comp ${ }^{-1}$ rule is similarly technical: to enable the proof of refutational completeness. In the following section we will try to justify its validity.

First let us notice that Equations (11) and (12) are rejected: it can be proved that from each of the formulas separately we can derive the rejected axiom in Equation (13). For Equation (11) to derive Equation (13) from it after substituting $R$ for $M$ we just need to strengthen the antecedent and weaken the consequent. In Equation (12) we first need to substitute $S$ for $P$ and $M$ for $S$. Then, using the fact, that $M i M \wedge M a S \wedge M a P \rightarrow S i P$ is a theorem of the system we can use classical proposition calculus to derive Equation (13). Thus, each Horn formula of the system is either a theorem or a rejected formula.

Now, we need to prove the same fact for the formulas of the form:

$$
\alpha \rightarrow \beta_{1} \vee \beta_{2}
$$

where $\alpha$ is a conjunction of atoms, and $\beta_{1}$ and $\beta_{2}$ are atoms. 
For this purpose it will be useful to note that the rejected axiom is equivalent to the following longer formula:

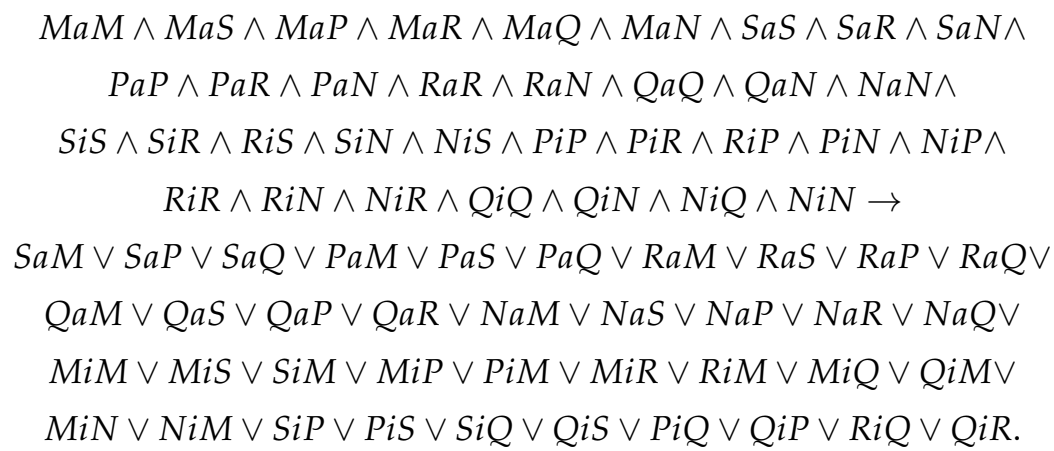

The intuitive meaning of the formula is not straightforward. It is a maximal combination of atoms built from six variables put on the both sides of the implications that does not allow one to derive consequent from antecedent, needed from the technical point of view to prove the completeness result.

The derivation from Equations (13) to (15) is valid since both the antecedent and consequent of Equation (13) are included in respectively the antecedent and the consequent of Equation (15). The derivation from Equation (15) to Equation (13) is based on the theorems of the system of the form of:

- implications with elements of the antecedent of Equation (15) not present in the antecedent of Equation (13) as the consequent and the antecedent of Equation (13) (or its fragment) as the antecedent, e.g.,

$$
M a S \wedge S a R \rightarrow M a R,
$$

- and implications with the elements of the consequent of Equation (15) not present in the consequent of Equation (13) and the antecedent of Equation (13) (or its fragments) as antecedents and elements of the antecedent of Equation (15) not present in the antecedent of Equation (13) as consequents, e.g.,

$$
S a M \wedge S i S \wedge M a S \wedge M a P \rightarrow S i P .
$$

Thus, all the elements of Equation (15) not included in Equation (13) can be eliminated.

Now we can move on to the main point of this part of the proof: the analysis of all possible forms of Equation (14). Its consequent may take one of the three following forms (with possibly different variables): (i) $S i P \vee R i Q$, (ii) $S i P \vee R a Q$, (iii) $S a P \vee R a Q$.

In case (i), any formula of the discussed shape $\alpha \rightarrow \operatorname{SiP} \vee R i Q$ is a theorem if $\alpha \rightarrow \operatorname{SiP}$ or $\alpha \rightarrow R i Q$ is a theorem. In case (ii), a formula is a theorem when one of the following conditions is fulfilled:

- $\alpha \rightarrow \operatorname{SiP}$ is a theorem,

- $\alpha \rightarrow R a Q$ is a theorem,

- $\quad$ the following conditions are satisfied: (I) $\alpha$ contains a chain connecting $R$ with $S$ (or in the place of both $S$ and $R$ the same variable occurs), and (II) $\alpha$ contains a chain connecting $R$ with $P$ (or in the place of both $P$ and $R$ the same variable occurs).

In case (iii), any formula of the discussed shape $\alpha \rightarrow S a P \vee R a Q$ is a theorem if $\alpha \rightarrow S a P$ or $\alpha \rightarrow R a Q$ is a theorem.

In all cases (i)-(iii), if a formula is not the theorem described above, after renaming the variables when needed, it contain only elements of the antecedent of Equation (15) in the antecedent and only elements of the consequent of Equation (15) in the consequent. All formulas fulfilling this condition are rejected. Thus, each formula of the shape seen in Equation (14) is a theorem or is rejected. 
It remains to prove that the set of theorems and the set of rejected formulas are disjoint. For that we need to show (a) that the rules of rejection lead from non-theorems to other non-theorems and (b) that the rejected axiom is not a theorem.

As for (a) since for rules $M P^{-1}$ and $S u b^{-1}$ that fact is obvious, the interesting case is the weak version of $\operatorname{Comp}^{-1}$. Here we need to show that if a formula $\alpha \rightarrow \beta_{1} \vee \beta_{2} \vee \beta_{3}$, where $\alpha$ is a conjunction of atoms and $\beta_{1}, \beta_{2}$ and $\beta_{3}$ are atoms, is a theorem of the system, then at least one of the $\alpha \rightarrow \beta_{i} \vee \beta_{j}$ $(i, j \in\{1,2,3\})$ is also a theorem. The proof of that fact is based on the constatation that the only non-Horn axiom of the system in Equation (10) cannot be effectively used twice in any derivation in the system, so the only way to obtain a formula of the form $\alpha \rightarrow \beta_{1} \vee \beta_{2} \vee \beta_{3}$ is by adding a new element of the consequent on the basis of the appropriate law of the classical propositional calculus.

To show that the rejected axiom is not a theorem of the system we can use the following matrices:

\begin{tabular}{|c|c|c|c|c|c|c|}
\hline$a$ & $n_{1}$ & $n_{2}$ & $n_{3}$ & $n_{4}$ & $n_{5}$ & $n_{6}$ \\
\hline$n_{1}$ & 1 & 1 & 1 & 1 & 1 & 1 \\
\hline$n_{2}$ & 0 & 1 & 0 & 1 & 0 & 1 \\
\hline$n_{3}$ & 0 & 0 & 1 & 1 & 0 & 1 \\
\hline$n_{4}$ & 0 & 0 & 0 & 1 & 0 & 1 \\
\hline$n_{5}$ & 0 & 0 & 0 & 0 & 1 & 1 \\
\hline$n_{6}$ & 0 & 0 & 0 & 0 & 0 & 1 \\
\hline
\end{tabular}

\begin{tabular}{|c|c|c|c|c|c|c|}
\hline$i$ & $n_{1}$ & $n_{2}$ & $n_{3}$ & $n_{4}$ & $n_{5}$ & $n_{6}$ \\
\hline$n_{1}$ & 0 & 0 & 0 & 0 & 0 & 0 \\
\hline$n_{2}$ & 0 & 1 & 0 & 1 & 0 & 1 \\
\hline$n_{3}$ & 0 & 0 & 1 & 1 & 0 & 1 \\
\hline$n_{4}$ & 0 & 1 & 1 & 1 & 0 & 1 \\
\hline$n_{5}$ & 0 & 0 & 0 & 0 & 1 & 1 \\
\hline$n_{6}$ & 0 & 1 & 1 & 1 & 1 & 1 \\
\hline
\end{tabular}

The matrices indicate the truth values of atoms when values $n_{i}(1 \leq i \leq 6)$ are substituted for nominal variables in formulas. Checking that Shepherdson's axioms receive always the value 1 is a usual routine. Rejected axiom in Equation (13) receives the value 0 when we put $n_{1}$ for $M, n_{2}$ for $S, n_{3}$ for $P, n_{4}$ for $R, n_{5}$ for $Q$ and $n_{6}$ for $N$.

\subsection{Refutation and Adequacy}

Let us stress that in the presentation of systems in the previous section we did not mention at all set-theoretical models of categorical sentences. That allows us to see syllogistic as a theory on its own, largely independent from the set-theoretical intuitions that are contemporarily usually applied in order to understand syllogistic.

The refutationaly complete axiomatic presentation of syllogistic may be an alternative way to control the correctness of the formalization. To check that a system is correct we need to show that axioms are intuitively correct and rejected axioms are not, and that rules used to deduce theorems from axioms and rejected formulas from rejected axioms (and theorems) work properly. Since correctness is not with respect to another formal system (like a set of models) the argumentation here cannot be strictly formal.

As for axioms of the three systems let us first look at their common part consisting of axioms in Equations (3) and (4). They come from Aristotle and seem to be intuitively very clear and convincing. The remaining parts of the system have much to do with empty names. In Łukasiewicz's system empty names are not allowed. Thus, if we only accept that using the same name twice in positive categorical sentences, like $S a S$ and $S i S$, makes sense at all, we should also accept that such sentences are true. In the case of Lemmon's system both specific axioms in Equations (6) and (7) express the core of the strong interpretation of $S a P$ in combination with the existential commitment of $i$. In the case of Shepherdson's system we got $S a S$ accepted in the axiom in Equation (1) as the weak interpretation of $a$ makes it true also for empty names. Equation (9) similarly to Equation (7) is based on the existential commitment of $i$. Finally, Equation (10) expresses the fact that a name $S$ is either empty (then $S a P$ has to be true under the weak interpretation of $a$ ) or non-empty (then SiS is true). Thus, we can say that the axioms of each system correctly reflect their background intuitions.

To show that a rejected axiom should be rejected it is enough to find a counterexample since we just need to confirm that it is not valid. That is the way that Aristotle worked. Let us look at the formulas used as rejected axioms in the axiomatic systems of syllogistic we have discussed. 
For Equation (5) we can take a cat for $S$, a dog for $P$ and an animal for $M$. Both dogs and cats are animals but no cat is a dog. For Equation (8) we can take a cat for $P$ and a unicorn for $S$ (assuming that unicorns do not exist and the sentence 'some unicorns are unicorns' is therefore false). Even if every cat is a cat it is not the case that some unicorns are unicorns. As Equation (13) is more complicated the counterexample for it is a bit more difficult to follow. Let us put a unicorn for $M$, a cat for $S$, a $\operatorname{dog}$ for $P$, a mammal for $R$, a parrot for $Q$ and an animal for $N$. Then all the elements of the antecedent of Equation (13) are true: every unicorn is a cat (since there are no unicorns), every unicorn is a dog, every unicorn is a parrot, every cat is a mammal, every dog is a mammal, every mammal is an animal, every parrot is an animal, some cats are cats, some dogs are dogs and some parrots are parrots, but neither any cat is a dog nor any mammal is a parrot.

The rules $M P$ and $S u b$, and their refutational counterparts $M P^{-1}$ and $S u b^{-1}$ seem to be very natural and common in logic. The justification of decomposition rules is less obvious. In its stronger version Comp ${ }^{-1}$ reflects the simplicity of syllogisms as single-conclusion schemata giving unequivocal result. It's modified version Comp $p_{2}^{-1}$ is weaker, because the Shepherdson's system has Equation (10) as an axiom and, because of this, $\operatorname{Comp}^{-1}$ is not valid there. Thus, the system allows for two alternative conclusions of a syllogism but no more than two. This principle can be understood as acknowledging Equation (10) and its consequences as an exception, which is limited by Comp $p_{2}^{-1}$.

To sum up, all conditions required for the discussed systems to be a correct representation of the intuitions they formalize are fulfilled. That gives us an example of how to discuss the correctness (soundness and completeness) of logic without models on the basis of refutation techniques having their sources in Aristotelian logic.

\section{Conclusions}

Aristotle and contemporary logicians share the same aspiration to give intuitions about correct reasoning a precise formulation. We have presented how Łukasiewicz's ideas of an axiomatic refutation system, inspired by Aristotle, work as a tool of assessment of such a formulation. In particular we have discussed the issue of correctness of three axiomatic systems of syllogistic introduced by Łukasiewicz, Lemmon and Shepherdson.

Let us point out the main conclusions of these considerations. Firstly, we should acknowledge that syllogistic is a fully-fledged deductive system and that Aristotle conducted interesting metalogical studies concerning it. Secondly, when investigating modern reconstructions of syllogistic, it is more interesting, in my opinion, to look at the content of the theories than at the particular tools of formalization. Thirdly, to assess the quality of the content of a theory, including such reconstructions, the most important feature is their adequacy consisting of correctness and completeness. Axiomatic refutation is an interesting method of showing adequacy. It can be used as an alternative to the model-theoretical approach commonly used in contemporary logic.

Finally, as for the three systems of Łukasiewicz's style syllogistic considered within the paper we can see that it is possible to construct refutation counterparts for all of them. All three refutational formalizations allow us to show adequacy of the systems with respect to the intuitions they follow. However, for Shepherdson's system which includes an axiom in a form of disjunction of atomic formulas, the refutation system itself and the proof of adequacy are much more complicated.

Funding: The project is funded by the Minister of Science and Higher Education within the program under the name "Regional Initiative of Excellence" in 2019-2022, project number: 028/RID/2018/19, the amount of funding: 11742500 PLN.

Acknowledgments: I would like to thank Urszula Wybraniec-Skardowska for discussions about refutation and encouragement to prepare this paper, anonymous reviewers for their valuable comments and Zdzisław Dywan, who has introduced me to the realm of Aristotelian logic.

Conflicts of Interest: The author declare no conflict of interest. 


\section{References}

1. Smith, R. Aristotle's Logic. In The Stanford Encyclopedia of Philosophy; Zalta, E.N., Ed.; Metaphysics Research Lab, Stanford University: Stanford, CA, USA, 2019.

2. Glashoff, K. Aristotelian Syntax from a Computational-Combinatorial Point of View. J. Log. Comput. 2005, 15, 949-973. [CrossRef]

3. Steinkrüger, P. Aristotle's assertoric syllogistic and modern relevance logic. Synthese 2015, 192, $1413-1444$. [CrossRef]

4. Crager, A. Meta-Logic in Aristotle's Epistemology. Ph.D. Thesis, Princeton University, Princeton, NJ, USA, 2015.

5. Read, S. Aristotle's Theory of the Assertoric Syllogism. Available online: https://philarchive.org/archive/ REAATO-5 (accessed on 1 April 2020).

6. Moss, L. Completeness theorems for syllogistic fragments. In Logics for Linguistic Structures; Hamm, S.K., Ed.; Mouton de Gruyter: Berlin, Germany; New York, NY, USA, 2008; pp. 143-174.

7. Pratt-Hartmann, I.; Moss, L. Logics for the relational syllogistic. Rev. Symb. Log. 2009, 2, 1-37. [CrossRef]

8. Glashoff, K. An Intensional Leibniz Semantics for Aristotelian Logic. Rev. Symb. Log. 2010, 3, $262-272$. [CrossRef]

9. Moss, L.S. Syllogistic Logics with Verbs. J. Log. Comput. 2010, 20, 947-967. [CrossRef]

10. Kulicki, P. On a Minimal System of Aristotle's Syllogistic. Bull. Sect. Log. 2011, 40, 129-145.

11. Moss, L.S. Syllogistic Logic with Comparative Adjectives. J. Log. Lang. Inf. 2011, 20, 397-417. [CrossRef]

12. Rini, A. Aristotle's Modal Proofs. Prior Analytics A8-22 in Predicate Logic; Springer: Berlin, Germany, 2011.

13. Kulicki, P. On minimal models for pure calculi of names. Log. Log. Philos. 2012, 1, 1-16. [CrossRef]

14. Bellucci, F.; Moktefi, A.; Pietarinen, A. Diagrammatic Autarchy: Linear diagrams in the 17th and 18th centuries. In Proceedings of the First International Workshop on Diagrams, Logic and Cognition, Kolkata, India, 28-29 October 2012; Burton, L.C., Ed.; CEUR Workshop Proceedings: Kolkata, India, 2013.

15. Pratt-Hartmann, I. The Syllogistic with Unity. J. Philos. Log. 2013, 42, 391-407. [CrossRef]

16. Castro-Manzano, J. Re(dis)covering Leibniz's Diagrammatic Logic. Tópicos Revista de Filosofía 2017, 52, 89-116.

17. Pietruszczak, A.; Jarmużek, T. Pure Modal Logic of Names and Tableau Systems. Stud. Log. 2018, 106, 1261-1289. [CrossRef]

18. Sautter, F.T.; Secco, G.D. A Simple Decision Method for Syllogistic. In Proceedings of the Diagrammatic Representation and Inference-10th International Conference, Diagrams 2018, Edinburgh, UK, 18-22 June 2018. Lecture Notes in Computer Science; Chapman, P., Stapleton, G., Moktefi, A., Pérez-Kriz, S., Bellucci, F., Eds.; Springer: Berlin, Germany, 2018; Volume 10871, pp. 708-711. [CrossRef]

19. Endrullis, J.; Moss, L.S. Syllogistic logic with “Most”. Math. Struct. Comput. Sci. 2019, 29, 763-782. [CrossRef]

20. Bocheński, I.M. Ancient Formal Logic; North-Holland: Oxford, UK, 1951.

21. Aristotle. Prior Analytics. Book I; Translated with an Introduction and Commentary by Gisela Striker; Clarendon Press: Oxford, UK, 2014.

22. Bocheński, I.M. The Methods of Contemporary Thought; D. Reidel: Dordrecht, The Netherlands, 1965.

23. Lear, J. Aristotle's compactness proof. J. Philos. 1979, 76, 198-215. [CrossRef]

24. Scanlan, M. On finding compactness in aristotle. Hist. Philos. Log. 1983, 4, 1-8. [CrossRef]

25. Smiley, T. What is a syllogism? J. Philos. Log. 1973, 2, 136-154. [CrossRef]

26. Boger, G. Completion, reduction and analysis: Three proof-theoretic processes in Aristotle's Prior Analytics. Hist. Philos. Log. 1998, 19, 187-226. [CrossRef]

27. Adžic, M.; Došen, K. Gödel's Notre Dame Course. Bull. Symb. Log. 2016, 22, 469-481. [CrossRef]

28. Corcoran, J. Completeness of an ancient logic. J. Symb. Log. 1972, 37, 696-702. [CrossRef]

29. Corcoran, J. Aristotle's Natural Deduction System. In Ancient Logic and Its Modern Interpretations; Reidel Publishing Co.: Dordrecht, The Netherlands, 1974; pp. 1-100.

30. Frege, G. Sense and Reference. Philos. Rev. 1948, 57, 209-230. [CrossRef]

31. Słupecki, J. Uwagi o sylogistyce Arystotelesa. Ann. UMCS 1946, I, 187-191.

32. Wedberg, A. The Aristotelian theory of classes. Ajutas 1948, 15, 299-314.

33. Menne, A. Logik und Existenz; Westkulturverlag Anton Hain: Berlin, Germany, 1954.

34. Shepherdson, J. On the Interpretation of Aristotelian Syllogistic. J. Symb. Log. 1956, 21, 137-147. [CrossRef] 
35. Geach, P.T. History of the Corruption of Logic (1968). In Logic Matters; University of California Press: Berkeley, CA, USA, 1980; pp. 44-61.

36. Ajdukiewicz, K. Logika Pragmatyczna; PWN: Warszawa, Poland, 1965.

37. Borkowski, L. Logika Formalna; PWN: Warszawa, Poland, 1970.

38. Grzegorczyk, A. Zarys Logiki Matematycznej; PWN: Warszawa, Poland, 1969.

39. Pogorzelski, W.A. Elementarny Stownik Logiki Formalnej; Dział Wydawnictw Filii Uniwersytetu Warszawskiego: Białystok, Poland, 1992.

40. Marciszewski, W. (Ed.) Mała Encyklopedia Logiki; PWN: Warszawa, Poland, 1988.

41. Waragai, T.; Oyamada, K. A System of Ontology Based on Identity and Partial Ordering as an Adequate Logical Apparatus for Describing Taxonomical Structures of Concepts. Ann. Jpn. Assoc. Philos. Sci. 2007, 15, 123-149. [CrossRef]

42. Słupecki, J.; Bryll, G.; Wybraniec-Skardowska, U. Theory of rejected propositions. I. Stud. Log. 1971, 29, 75-115. [CrossRef]

43. Goranko, V.; Pulcini, G.; Skura, T. Refutation Systems: An Overview and Some Applications to Philosophical Logics. In Knowledge, Proof and Dynamics; Liu, F., Ono, H., Yu, J., Eds.; Springer: Singapore, 2020; pp. 173-197.

44. Wybraniec-Skardowska, U. Rejection in Łukasiewicz's and Słupecki's Sense. In The Lvov-Warsaw School. Past and Present; Garrido, Á., Wybraniec-Skardowska, U., Eds.; Springer International Publishing: Cham, Switzerland, 2018; pp. 575-597. [CrossRef]

45. Kulicki, P. The Use of Axiomatic Rejection. In The Logica Yearbook 1999; Childers, T., Ed.; Filosofia: Prague, Czech Republic, 2000; pp. 109-117.

46. McKinsey, J. The Decision Problem for some Classes of Sentences without Quantifiers. J. Symb. Log. 1943, 8, 61-76. [CrossRef]

47. Słupecki, J. Z badań nad sylogistyka Arystotelesa; Wrocławskie Towarzystwo Naukowe: Wrocław, Poland, 1948.

48. Lukasiewicz, J. Aristotle's Syllogistic from the Standpoint of Modern Formal Logic; Clarendon Press: Oxford, UK, 1952.

49. Lemmon, E.J. Quantifiers and Modal Operators. Proc. Aristot. Soc. 1958, 58, 245-268. [CrossRef]

50. Kulicki, P. Aksjomatyczne Systemy Rachunku Nazw; Wydawnictwo KUL: Warszawa, Poland, 2011.

51. Prior, A.N. Formal Logic; Clarendon Press: Oxford, UK, 1962.

(C) 2020 by the authors. Licensee MDPI, Basel, Switzerland. This article is an open access article distributed under the terms and conditions of the Creative Commons Attribution (CC BY) license (http://creativecommons.org/licenses/by/4.0/). 